\title{
Erratum to: A comparison of magnetic resonance arthrography and arthroscopic findings in the assessment of anterior shoulder dislocations
}

\author{
R. W. Jordan ${ }^{1} \cdot$ R. Naeem ${ }^{1} \cdot$ K. Srinivas ${ }^{1} \cdot$ G. Shyamalan ${ }^{1}$
}

Published online: 30 April 2015

(C) ISS 2015

Erratum to: Skeletal Radiol (2015) 44:653-657

DOI 10.1007/s00256-014-2080-6

The original version of this article unfortunately contained errors. Several occurrences of "Magnetic resonance arthrography" (MRA) were inadvertently replaced by "Magnetic resonance angiography".

\footnotetext{
R. W. Jordan

Robert.jordan@doctors.org.uk

1 Birmingham Heartlands Hospital, Bordesley Green East,

Birmingham B9 5SS, UK
} 